\title{
Preliminary in vivo Investigation into the Relationship between Canal Preparation Protocol and Incidence of Postoperative Pain in Single-visit and Two-visit Endodontic Therapy: A Randomized Controlled Clinical Trial
}

${ }^{1}$ Sumita Bhagwat, ${ }^{2}$ Nehal Sheth, ${ }^{3}$ Leena Padhye

\begin{abstract}
Introduction: Postoperative pain is a major concern for patients undergoing root canal treatment. Much research has gone into the alteration of several variables to determine the elusive technique that will result in no postoperative pain.
\end{abstract}

Aim: The aim of our study was to investigate the incidence, severity, and duration of postoperative pain in relation to technique of root canal preparation and compare the difference in incidence, severity, and duration of postoperative pain in single- and two-sitting root canal therapy when different instrumentation techniques are used.

Materials and methods: Sixty adult patients requiring root canal therapy were selected and divided into six groups. Singlesitting root canal treatment was carried out in three groups, with three different canal preparation methods (group I Conventional hand files, group II - hand ProTaper files, and group III - rotary ProTaper files). Two-sitting root canal treatment was carried out in three groups, with the same canal preparation methods (group IV - conventional hand files, group V hand ProTaper files, and group VI - rotary ProTaper files). Postoperatively, the subjects were instructed to fill out a series of self-report questionnaires for pain incidence after 1 day, 2 days, 3 days, 1 week, and 2 weeks. After 2 weeks, the patients were recalled and their responses were duly noted.

Results: Results were tabulated and statistically analyzed using Mann-Whitney U test.

Conclusion: There was a significant difference in incidence, character, and duration of postoperative pain in rotary-prepared canals as compared with hand-prepared canals in both singleand two-sitting endodontics.

Clinical significance: The purpose of this study was to search for a predictability in incidence, duration, and character of postoperative pain in canals instrumented with hand and rotary

${ }^{1}$ Professor and Postgraduate Guide, ${ }^{2}$ Ex-resident, ${ }^{3}$ Professor and Head

${ }^{1-3}$ Department of Conservative Dentistry and Endodontics, DY Patil University School of Dentistry, Navi Mumbai, Maharashtra India

Corresponding Author: Sumita Bhagwat, Professor and Postgraduate Guide, Department of Conservative Dentistry and Endodontics, DY Patil University School of Dentistry, Navi Mumbai, Maharashtra, India, Phone: +912221713763, e-mail: sumita1572@gmail.com instruments. Knowing this will enable clinicians to make more prudent choices in instruments, techniques, and protocols for root canal therapy.

Keywords: Manual preparation, Pain duration, Postendodontic pain, Postoperative pain, Rotary preparation.

How to cite this article: Bhagwat S, Sheth N, Padhye L. Preliminary in vivo Investigation into the Relationship between Canal Preparation Protocol and Incidence of Postoperative Pain in Single-visit and Two-visit Endodontic Therapy: A Randomized Controlled Clinical Trial. J Contemp Dent 2017;7(1):48-52.

Source of support: Nil

Conflict of interest: None

\section{INTRODUCTION}

In the past decade, the shift from manual canal preparation to rotary canal preparation during endodontic therapy has been noteworthy. But even with the use of rotary methods, postoperative pain continues to appear with high frequency. ${ }^{1,2}$ Much research has gone into the alteration of variables like interappointment time $\operatorname{span}^{3}$ and number of sittings to reduce postoperative pain. Till date, there is very little research that compares postoperative pain following hand vs rotary canal preparation techniques. Our study aimed at testing the hypothesis that there is a definite relationship between occurrence of postoperative pain and the technique used for canal preparation. This clinical study has compared the incidence, duration, and character of postoperative endodontic pain in canals prepared with hand instruments ( $2 \%$ and hand ProTaper $\left.{ }^{\mathrm{TM}}\right)$ and those prepared with rotary instrumentation (ProTaper ${ }^{\mathrm{TM}}$ ) in both single- and two-sitting endodontics.

\section{MATERIALS AND METHODS}

Sixty adult patients aged between 16 and 65 years, requiring root canal therapy in anterior and singlerooted premolar teeth were selected from the Department of Conservative Dentistry and Endodontics for this study. 


\section{Selection Criteria}

- Asymptomatic vital/nonvital teeth requiring root canal treatment

- Teeth with sound periodontal apparatus

- Teeth without pus or inflammatory exudate draining through the canal

- Teeth without anatomic variations

- Patients not on analgesics or sedative medication prior to root canal therapy

- Teeth without sinus tract

- Teeth without history of trauma

- Teeth selected as abutments or to be retained under an overdenture

- Teeth with periapical radiolucency not exceeding $3 \times 3 \mathrm{~mm}$ in size

Patients with any systemic diseases, pregnant patients, immunocompromised patients, retreatment cases, and teeth with calcified canals were not included in the study. At an initial appointment, subjects were informed about the nature of the study. A thorough clinical examination including vitality testing and case history was taken. A preoperative radiograph was taken to check the canal, periodontal tissues, and presence of periapical radiolucency. Written consent was obtained from each patient prior to the study. Approval was obtained from the ethical committee of the institute and from the scientific research committee of the university, and permissions were obtained for the conduction of the study. The postoperative pain rating was done in accordance with a benchmark study by Wang et al. ${ }^{4}$

The subjects were randomly divided into six groups: Group I: Ten teeth treated in single visit using conventional hand files for instrumentation of the canal Group II: Ten teeth treated in single visit using hand ProTaper files for instrumentation of the canal

Group III: Ten teeth treated in single visit using rotary ProTaper files for instrumentation of the canal

Group IV: Ten teeth treated in two visits with interappointment time of 72 hours using conventional hand files for instrumentation of the canal

Group V: Ten teeth treated in two visits with interappointment time of 72 hours using hand ProTaper files for instrumentation of the canal.

Group VI: Ten teeth treated in two visits with interappointment time of 72 hours using rotary ProTaper files for instrumentation of the canal.

Local anesthesia was administered and root canal treatment was initiated. The tooth was isolated using rubber dam. Caries/defective restoration was removed. Root canal treatment was completed as per instrumentation technique and obturation schedule of each group. Obturation was completed by lateral condensation technique with standardized commercially available gutta-percha. ${ }^{5}$ Immediately following obturation of the tooth, each subject was instructed to maintain a record of pain incidence for the next 2 weeks. The record included a note of pain following 1 day, 2 days, 3 days, 1 week, and 2 weeks after obturation. After 2 weeks, the patients were recalled and their responses were duly noted.

\section{Rating for Pain ${ }^{4}$}

- 0 - No pain

- 1 - Mild pain: any discomfort that did not require medication or emergency treatment, no matter how long it lasted

- 2 - Moderate pain: pain requiring medication

- 3 -Severe pain: pain that was not relived by medication and required palliative treatment

\section{RESULTS}

Table 1 gives the results of the pain responses reported by the patients of all the groups.

\section{Statistical Analysis of the Data}

After obtaining the responses from the subjects and tabulation of the same (Table 1), statistical analysis of the data was performed. Mann-Whitney U test (Table 2) was performed on the data obtained and interpretations and inferences were drawn from the same.

\section{DISCUSSION}

Postoperative pain in root canal treatment is more the norm than the oddity, and often the only differences are the severity and the duration of the same. Many different methods and instruments of canal preparation have come and gone but the only new technique here to stay is surely the rotary method of root canal preparation. There are several studies in the literature that state that postoperative pain is greater in single-sitting endodontics as compared with the two-sitting protocol, ${ }^{1,2,6}$ but there is no investigation into the difference between postoperative pain in hand-instrumented and rotary-instrumented canals.

In our study, comparison of the data (Table 1) showed that both single-sitting hand-prepared groups (groups I and II) showed a similar incidence of postoperative pain and it was of a short duration. Most patients were pain free 3 days after obturation. Comparison for significance by Mann-Whitney U test (Table 2 ) between group I ( $2 \%$ hand-prepared canals) and group II (Hand ProTaper ${ }^{\mathrm{TM}}$ prepared canals) did not show a significant difference. The incidence of postoperative pain in group III (rotary ProTaper ${ }^{\mathrm{TM}}$-prepared canals) was similar to groups I and II, 
Table 1: Data distribution for the pain level

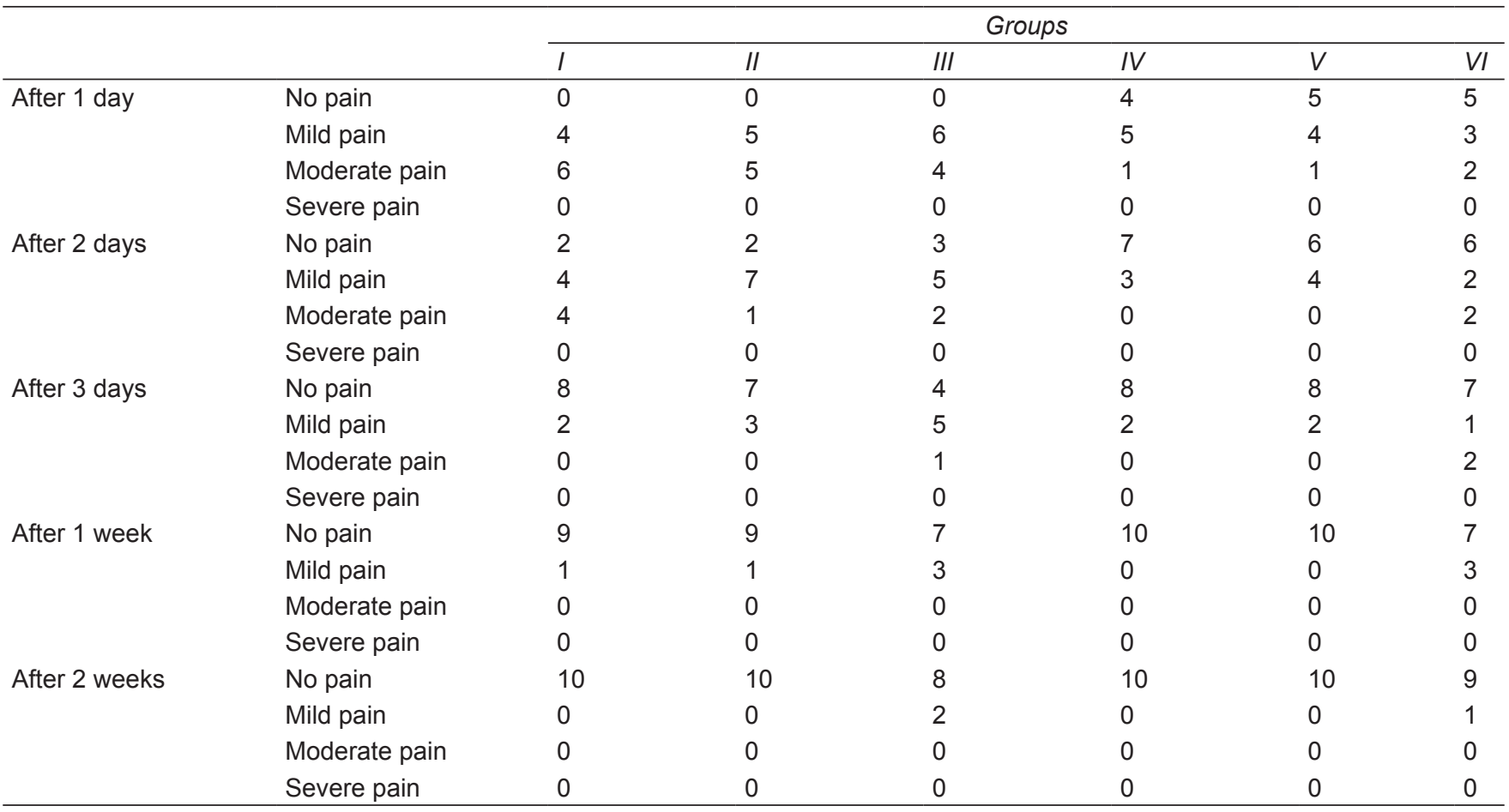

Table 2: Mann-Whitney U test result

\begin{tabular}{lll}
\hline Group I & Group J & -value \\
\hline I & II & 0.796 \\
& III & 0.353 \\
& IV & 0.063 \\
& V & 0.105 \\
& VI & 0.280 \\
II & III & 0.436 \\
& IV & $0.039^{*}$ \\
& V & $0.06^{*}$ \\
& VI & 0.230 \\
III & IV & $0.019^{*}$ \\
& V & $0.019^{*}$ \\
& VI & 0.123 \\
IV & V & 0.904 \\
& VI & 0.853 \\
V & VI & 0.739 \\
\hline *p-Value
\end{tabular}

${ }^{*} \mathrm{p}$-value $<0.05$ indicates significant difference

but the duration of the pain was significantly longer. The severity of postoperative pain in groups I to III was similar and there was no statistical significance in the difference ( $p$ > 0.05).

The incidence of postoperative pain in groups IV to VI (two-sitting groups) was half of groups I to III (singlesitting groups), and the difference in incidence of pain in the two-sitting groups was statistically significant $(\mathrm{p}<0.05)$ from the single-sitting groups, as statistically derived in Table 2.

Groups IV to VI showed low incidence of postoperative pain, and the difference among the groups was not statistically significant $(p>0.05)$. It was observed that compared with groups IV and V, the severity of postoperative pain in group VI was higher and of a longer duration.

When comparing single-sitting and two-sitting handprepared canals (groups I and II compared with groups IV and V), there was a significant difference $(\mathrm{p}<0.05)$ in incidence and duration of postoperative pain. Groups I and II showed a significantly higher incidence and duration of pain as compared with groups IV and V. The severity of pain in groups I and II was also higher than groups IV and V, and this difference was significant $(\mathrm{p}<0.05)$, as statistically derived in Table 2 .

On comparison of group III (single-sitting rotaryprepared canals) and group VI (two-sitting rotaryprepared canals), it was observed that the incidence of pain was lower in group VI, but this difference was not statistically significant $(p>0.05)$. First day postoperatively, the severity of pain was quite higher in group III as compared with group VI, but the difference in duration of pain in both the groups was not significant $(p>0.05)$, as statistically derived in Table 2.

It is likely that rotary preparation of root canals causes significant extrusion of debris beyond the apex and induces an inflammatory reaction, which is significantly greater than when root canals are prepared by hand instrumentation. This increased extrusion and associated inflammatory reaction may be responsible for greater immediate postoperative pain in rotary-prepared root canals than in hand-prepared root canals. 
Rao et $\mathrm{al}^{7}$ found that there was no difference in postoperative pain between patients treated in only one appointment and patients treated in two appointments. The majority of patients in both groups reported no pain or only minimal pain after 7 days of treatment. But in their study, the biomechanical preparation in both the groups was done by rotary method. Similar conclusions have been drawn by Raju et al. ${ }^{8}$

Wong et al, ${ }^{9}$ in their exhaustive review, say that neither single-visit endodontic treatment nor multiple-visit treatment can guarantee the absence of postoperative pain. They found that postoperative pain in both single-visit and multiple-visit endodontic treatment is nearly the same with little variation in duration and intensity, but there is no mention of any significant relation in terms of the character of pain.

Bhagwat and Mehta, ${ }^{6}$ in their study, found that nonvital teeth with periapical radiolucency exhibited relatively less pain as compared with nonvital teeth without periapical radiolucency, but the pain continued in a significant percent of teeth even after 2 weeks. Pain incidence dropped significantly within a period of 1 day to 2 weeks in vital teeth and nonvital teeth without periapical radiolucency. There was a tendency for less incidence of significant pain after a single-visit root canal treatment in these groups.

Gambarini et al ${ }^{10}$ compared postoperative incidence of pain using three different nickel-titanium instrumentation techniques: a rotary crown-down technique using TF instruments (SybronEndo, Orange, CA), a reciprocating single-file technique using WaveOne instruments (Maillefer Dentsply, Ballaigues, $\mathrm{CH}$ ), and a novel instrumentation technique (TF Adaptive, SybronEndo, Orange, CA), using a unique, proprietary movement, combining reciprocation and continuous rotation. Their findings showed a statistically significant difference in incidence of postoperative pain between the WaveOne $(p=0.021)$ technique and the other two techniques. They concluded that the difference in postoperative pain can be mainly related to the different instrumentation techniques.

In a very recent study, Arias et $\mathrm{l}^{11}$ suggest that a higher incidence of postoperative pain should be expected after manual root canal preparation, which is not in agreement with the findings of our study. However, a second major finding of the study is that when present, postoperative pain after a rotary canal preparation is expected to last longer, which supports our findings quite precisely.

Several researchers have worked on various variables like irrigation techniques, ${ }^{12}$ obturating techniques, ${ }^{13}$ variation in intraappointment time period, ${ }^{3}$ and administration of different analgesics as pretreatment medication ${ }^{14}$ in an attempt to device the best treatment protocol that results in minimum postoperative endodontic pain.
Within the limits of human error and an unaccountability of differences in individual patients' tolerance to pain, we found a definite correlation between canal preparation technique and postoperative pain and thus our hypothesis was proved right. Further clinical studies are required to support the correlation between canal preparation technique and postoperative pain found in our study.

\section{CONCLUSION}

From our in vivo study, we draw the following conclusion:

- The incidence and severity of postoperative pain in single-sitting endodontics is nearly the same in manual-prepared and rotary-prepared canals, but the duration of the pain in rotary-prepared canals is significantly longer in duration.

- The incidence of postoperative pain in two-sitting endodontics is significantly lower than singlesitting endodontics irrespective of method of canal preparation.

- The incidence of postoperative pain in two-sitting endodontics is low in hand as well as rotary-prepared canals, but the duration and severity of the postoperative pain is higher in rotary-prepared canals as compared with hand-prepared canals.

- Single-sitting hand-instrumented canals show significantly higher incidence and duration of postoperative pain when compared with two-sitting handinstrumented canals. There is significant difference in the severity of the postoperative pain. Single-sitting hand-instrumented canals show greater incidence of more severe postoperative pain than two-sitting hand-instrumented canals.

- Single-sitting rotary-prepared canals show a tendency for higher incidence of postoperative pain as compared with two-sitting rotary-prepared canals. The severity of the pain on the first postoperative day is likely to be higher in single-sitting rotary-prepared canals, but the duration of the pain may not differ from two-sitting rotary-prepared canals.

\section{REFERENCES}

1. Su Y, Wang C, Ye L. Healing rate and post-obturation pain of single- versus multiple-visit endodontic treatment for infected root canals: a systematic review. J Endod 2011 Feb;37(2):125-132.

2. Singh S, Garg A. Incidence of post-operative pain after single visit and multiple visit root canal treatment: a randomized controlled trial. J Conserv Dent 2012 Oct;15(4):323-327.

3. Bhagwat SA, Patel A, Mandke LP. The effect of variation in inter-appointment time on the incidence of post-operative pain in single-visit versus two visit endodontics: an in vivo study. J Int Clin Dent Res Organ 2012 Jan-Dec;4(1):9-17. 
4. Wang C, Xu P, Ren L, Dong G, Ye L. Comparison of postobturation pain experience following one-visit and two-visit root canal treatment on teeth with vital pulps: a randomized controlled trial. Int Endod J 2010 Aug;43(8):692-697.

5. Ingle, J.; Bakland, L. Endodontics. 6th ed. Chapter 27. India: B.C. Decker, Elsevier; 2008. p. 877-991.

6. Bhagwat $S$, Mehta D. Incidence of post-operative pain following single visit endodontics in vital and non-vital teeth: an in vivo study. Contemp Clin Dent 2013 Jul;4(3):295-302.

7. Rao KN, Kandaswamy R, Umashetty G, Rathore VPS, Hotkar C, Patil BS. Post-obturation pain following one-visit and two-visit root canal treatment in necrotic anterior teeth. J Int Oral Health 2014 Apr;6(2):28-32.

8. Raju TB, Seshadri A, Vamsipavani B, Abhilash K, Subhash AV, Kumari KVH. Evaluation of pain in single and multi rooted teeth treated in single visit endodontic therapy. J Int Oral Health 2014 Feb;6(1):27-32.

9. Wong AW, Zhang $\mathrm{C}$, Chu $\mathrm{CH}$. A systematic review of nonsurgical single-visit versus multiple-visit endodontic treatment. Clin Cosmet Investig Dent 2014 May 8;6:45-56.

10. Gambarini G, Testarelli L, De Luca M, Milana V, Plotino G, Grande NM, Rubini AG, Al Sudani D, Sannino G. The influence of three different instrumentation techniques on the incidence of postoperative pain after endodontic treatment. Ann Stomatol (Roma) 2013 Mar 20;4(1):152-155.

11. Arias A, de la Macorra JC, Azabal M, Hidalgo JJ, Peters OA. Prospective case controlled clinical study of post-endodontic pain after rotary root canal preparation performed by a single operator. J Dent 2015 Mar;43(3):389-395.

12. Ramamoorthi S, Nivedhitha MS, Divyanand MJ. Comparative evaluation of postoperative pain after using endodontic needle and EndoActivator during root canal irrigation: a randomised controlled trial. Aust Endod J 2015 Aug;41(2): 78-87.

13. Alonso-Ezpeleta LO, Gasco-Garcia C, Castellanos-Cosano L, Martín-González J, López-Frías FJ, Segura-Egea JJ. Postoperative pain after one-visit root-canal treatment on teeth with vital pulps: comparison of three different obturation techniques. Med Oral Patol Oral Cir Bucal 2012 Jul;17(4): e721-e727.

14. Sethi P, Agarwal M, Chourasia HR, Singh MP. Effect of single dose pretreatment analgesia with three different analgesics on postoperative endodontic pain: a randomized clinical trial. J Conserv Dent 2014 Nov;17(6):517-521. 